\title{
A novel design of a contractible, tubular continuum manipulator
}

\author{
Zhiguang Xing, Pengyuan Wang, Jianwen Zhao \\ Mechanical Engineering \\ Harbin Institute of Technology \\ Weihai, Shandong Province, China \\ zhaojianwen@hit.edu.cn
}

\begin{abstract}
Continuum manipulators have advantages in narrow space detections and operations than the rigid-body robots. In this paper, we novel designed a continuum manipulator with contractible/extensive abilities that give the manipulator more agile and flexible motion than those without these. The robotic system is composed of the continuum deforming body, driving tendons, and a mechanism. To enhance the displacement accuracy of the tendons and compact the package, the mechanism was designed as a controlled winding roller with rotation and translation motion. A prototype of the robotic system was made to evaluate the motion ability of the proposed design.
\end{abstract}

Keywords-continuum manipulator, contractile mechanism, workspace analysis

\section{INTRODUCTION}

Continuum manipulators (CM) have good obstacle avoidance ability and passive compliance which come from their flexible mechanism with a big length-to-diameter ratio. They can serve surgical operations, detection of aircraft or diesel engines, as well as some other operations with narrow spaces. [1-6]

There are many types of the CM, which mainly include tendon-driven manipulator with flexible backbones [7, 8], pneumatic arms composed of soft materials [9], concentric tube manipulator with very big length-to-diameter ratio [10], and snake-like robots with many independent severing motors-driven joints [11], etc.

In this paper, we focus on the CM with contract (extend) abilities. Reviewed the literatures, four types of contractible $\mathrm{CM}$ would be introduced as follows. (1). Pneumatic-driven CM. [9] They usually have soft-body structures that embed textiles or the differentiated wall thickness to constrict the undesired deformation. Moreover, researchers have developed a novel inflated manipulator that can grow its body even up to 70 meters. $[12,13]$ The pneumatic-driven $\mathrm{CM}$ is powered by the pneumatic pressure so that they are often confused by the inadequate bending stiffness and difficult controllability. (2) Concentric tube CM [14-16]. These manipulators are composed of several very slim hyperplastic shape memory tube and form an extensible antenna-like structure. Each tube was mechanical programming bent, and the bending direction was controlled by their relative axial displacement and rotations. They can benefit to minimally invasive surgery with the follow-the-leader motions. (3) Magnetically extensible, tendon actuated continuum robot (METABot). [4, 17, 18] In this design, the magnetic disk is assembled by permanent magnets which can provide the repulsive force, and all of them can slide along with the central hyperelastic rod. (4) Mechanical spring-based tendon actuated CM. [19, 20] Compared to the METABot, the repulsive force generated by the elastic potential energy of a compressed mechanical spring rather than that from the permanent magnets. Similar to the METABot, the mechanical spring-based tendon actuated CM eases the control complexities because it has better kinematics precision than pneumatic-driven $\mathrm{CM}$ and has a more agile workspace than the concentric tube CM.

In this paper, we designed a contractible CM based on the contractible mechanical spring. Different from the related work in literature [20], we designed a CM which is actuated by the tendons whose bending stiffness can be neglected rather than the flexible rods used in [20]. More, the tendons continuously go through each turn of the spring rather than using separated links so that smaller curvature radius, bigger stiffness, and more smooth steering motion can be achieved, and the mechanical spring-based elastic skeleton enables the big hollow channel to transport like liquids, grippers, lasers, and cameras, etc. Also, our CM has 2 independent segments, causing better flexibility, more degrees of freedom, bigger workspace than that in [20]. The key contributions of this paper were listed as below:

(1) Mechanical design of the robotic system, including the actuating module, robotic body, and control system.

(2) Comparison of the workspace for the continuum manipulator with and without contractile capacity.

(3) Prototype and simple motion demonstration.

\section{DESIGN OF THE ROBOTIC SYSTEM}

The continuum manipulator system is composed by a robotic body, 8 tendons, and a driving mechanism. The robotic body has 2 segments, each one was driven by 4 tendons. Sheaths of the tendons enable the remote driving ability of the robotic body, as well as the decoupling of the kinematics between the two segments. The tendon-driving mechanism is actuated by 8 reduced stepper motors (The stepper motor outputs torque of $0.05 \mathrm{Nm}$, and the reducer ratio is 3.71), as Fig. 1 shows. For accuracy control of the tendons, as well as the compact design requirements of the mechanism, we choose a rolling mechanism to pull the tendons. The tendons were wound on the reel with slots so that the disorder of tendons can be avoided. The rotor that is connected to the stepper motor rotates the reel, and there is only relative 
sliding movement between the reel and the rotor, then the tendon on the reel was pulling. To make the tendon has no translating skew, the reel should translate as the required speed. Here we use a fixed screw to provide the required translation (the reel has an internal thread acted as the nut to mate with the fixed screw). Fig. 2 is the prototype of the robotic system. Notably, the continuum robotic body has 6
DoFs (Degrees of Freedom) but the tendon-driven mechanism has 8 motors. It is declared that the redundant 2 DoFs was prepared for future work on stiffness variation research. Without payload consideration, the 2 redundant DoFs should be passively following the motion of the deforming robotic body required.

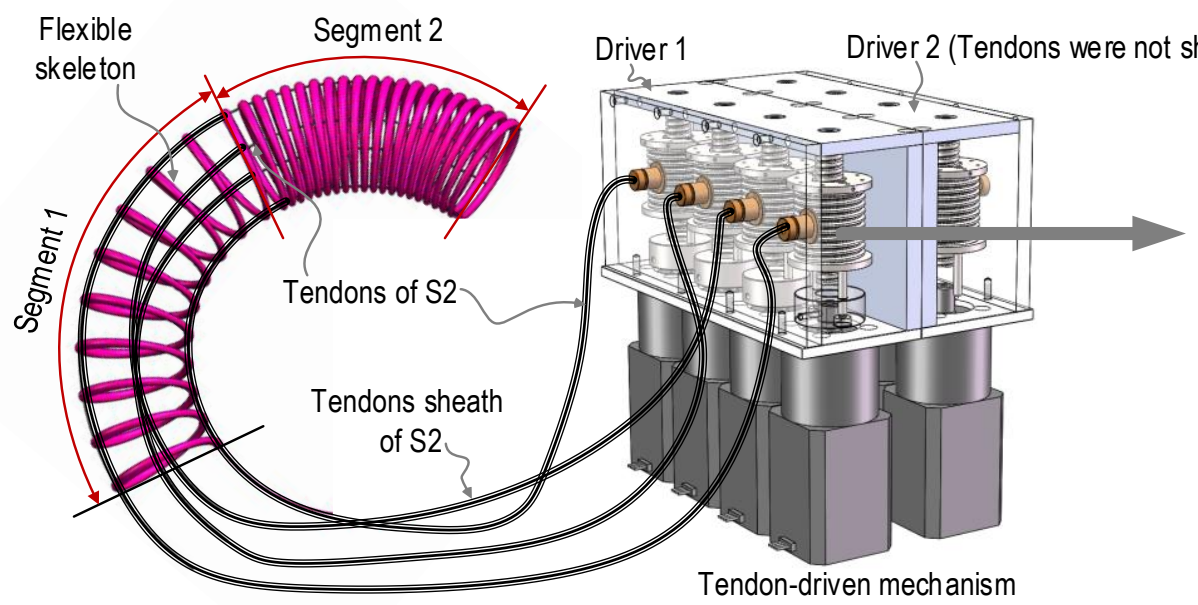

(a)

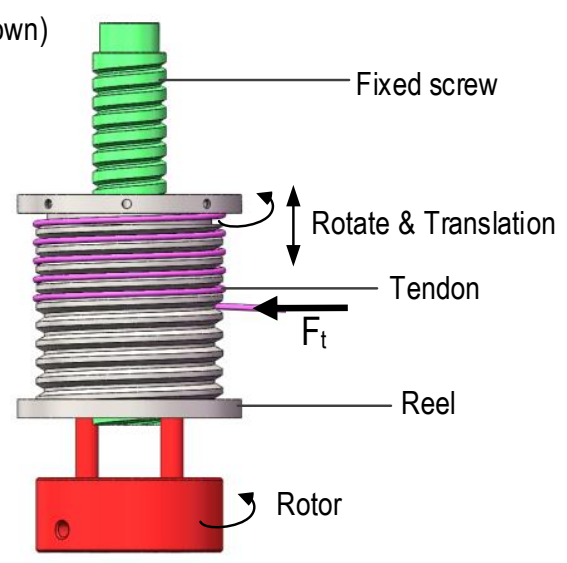

(b)

Fig. 1 The CAD model of the robotic system. Tendons and its sheaths of segment 1 were not shown in this figure (they similar to segment 2). (a). The CAD model of the system. (b). Tendon-rolling mechanism. The rotor rotates the reel which is matched with a

fixed screw, and the fixed screw gives the reel translation motion that guarantees the standing direction of the tendons.

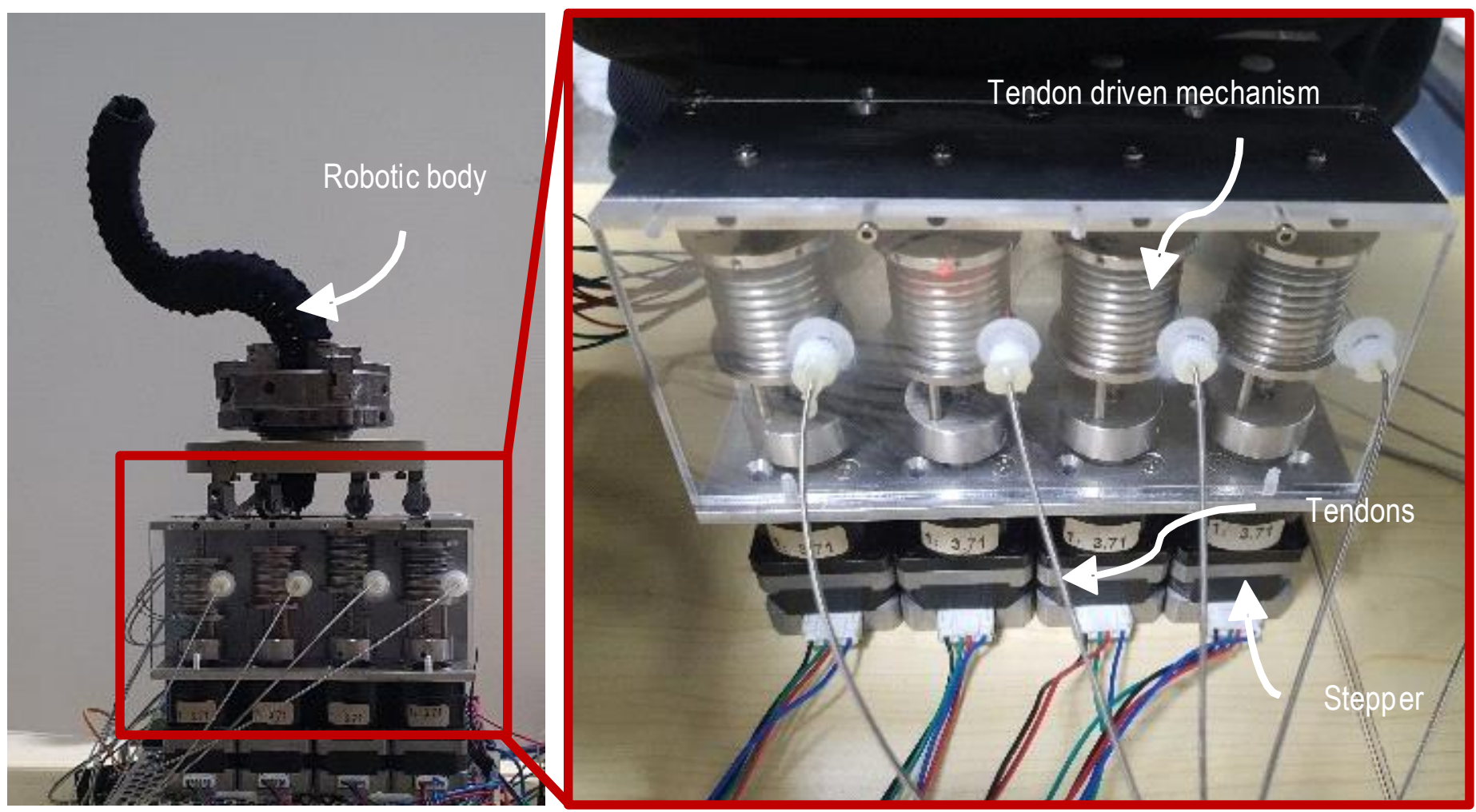

Fig. 2 The physical prototype of the robotic system. It is compromised by the robotic body and tendon driven mechanism, they are connected by the tendons (with sheaths). 


\section{WORKSPACE ANALYSIS OF THE MANIPULATOR}

In this section, we will analyze the workspace for the continuum manipulator with contractile ability compared to that without contractile ability.

Some assumptions should be declared for the contractile manipulator in advance: The central-axis of every segment remains a circular curve in deforming; the minimum length of the manipulator determined by the wire-diameter $d$ (in Fig. 3 ) of the spring-like flexible skeleton, and one embedded length of the tendon can keep in maximum meanwhile the other embedded tendon has been in shortest (i.e., the state that of $l_{1}=L_{0}, l_{2}=l_{\min }$ can be realized); the motion of the manipulator has geometric symmetry which makes its workspace is rotational symmetric volume so that we can ease the problem into a planar model.

Upon the assumptions, the characteristic parameters of the circular curve, those are bending angle theta and curvature radius rho in Fig. 3, can be calculated according to the below analyses.

The minimal length of the embedded tendon $l_{\min }=n d$, where $n$ is the turns of the flexible skeleton. The size parameters of our physical prototype were list in Table. 1.

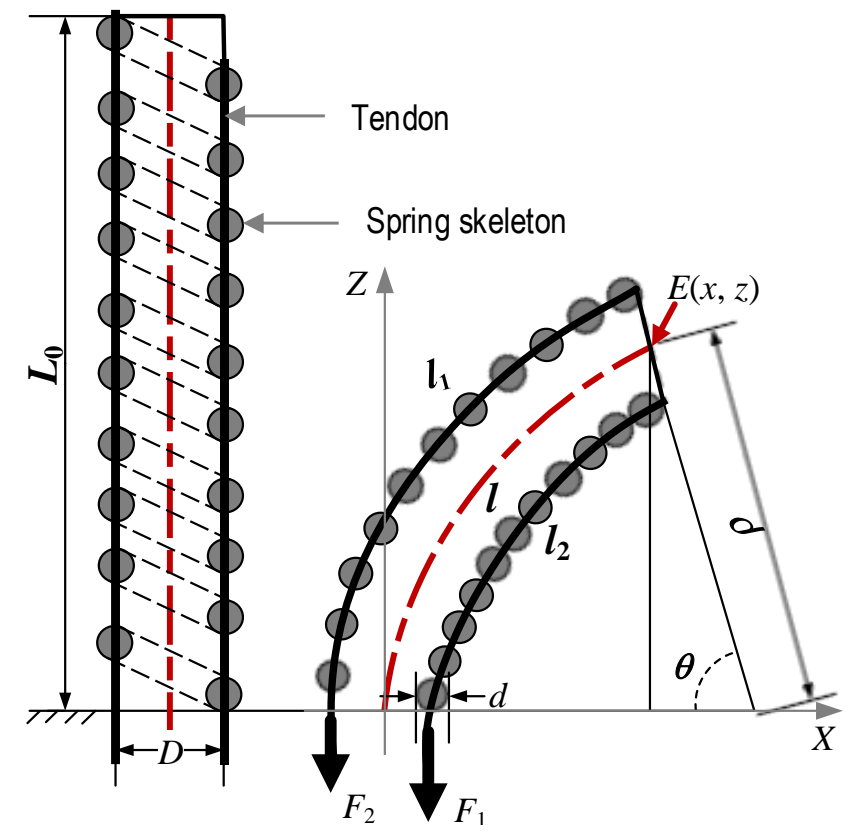

(a)

(b)

Fig. 3 The working principle of the robotic arm.

TABLE. 1 THE PARAMETERS OF THE PHYSICAL ROBOTICBODY.

\begin{tabular}{lll}
\hline \hline Symbol & & Value $(\mathrm{mm})$ \\
\hline$L_{0}$ & $\begin{array}{l}\text { The initial length of the embedded tendons } \\
\text { of a one-segment manipulator }\end{array}$ & 150 \\
$n$ & The turns of the spring-like flexible skeleton & 25 \\
$D$ & The nominal diameter of the robotic-body & 22 \\
$d$ & The diameter of the wire-diameter & 2 \\
\hline \hline
\end{tabular}

The geometry in Fig. 3 can yield that bending angle and curvature can be characterized as

$$
\left\{\begin{array}{l}
\rho=\left(l_{1}+l_{2}\right) D /\left[2\left(l_{1}-l_{2}\right)\right] \\
\theta=2\left(l_{1}-l_{2}\right) / D
\end{array}\right.
$$

The special situation of $l_{1}=l_{2}$ was not taken into account here. The end-point $E$ can be donated as

$$
\left\{\begin{array}{l}
x=\rho(1-\cos \theta) \\
y=\rho \sin \theta
\end{array}\right.
$$

First, we use a generic ergodicity algorithm to scan the workspace of a one-segment contractile continuum manipulator, as Fig. 4(a) shows. For comparing to the workspace of a one-segment manipulator without contractile ability, we use the same algorithm to obtain Fig. 4(b). The additional constraint compared to that with contractile capability is that the centerline of the robotic-body should be kept in the length of $L_{0}$ in the deforming process, i.e., $l_{\min }$ should be much more than $n h$, in this paper and $l_{1}+l_{2}=2 L_{0}$, we predefined that the maximal bending angle $\theta_{\max }=\pi$. (This is already an exaggerated contractile/extensile range according to the published works). The constraints were listed in (3)

$$
\left\{\begin{array}{l}
l_{1}+l_{2}=2 L_{0} \\
l_{1}-l_{2} \leqslant \pi D / 2
\end{array}\right.
$$

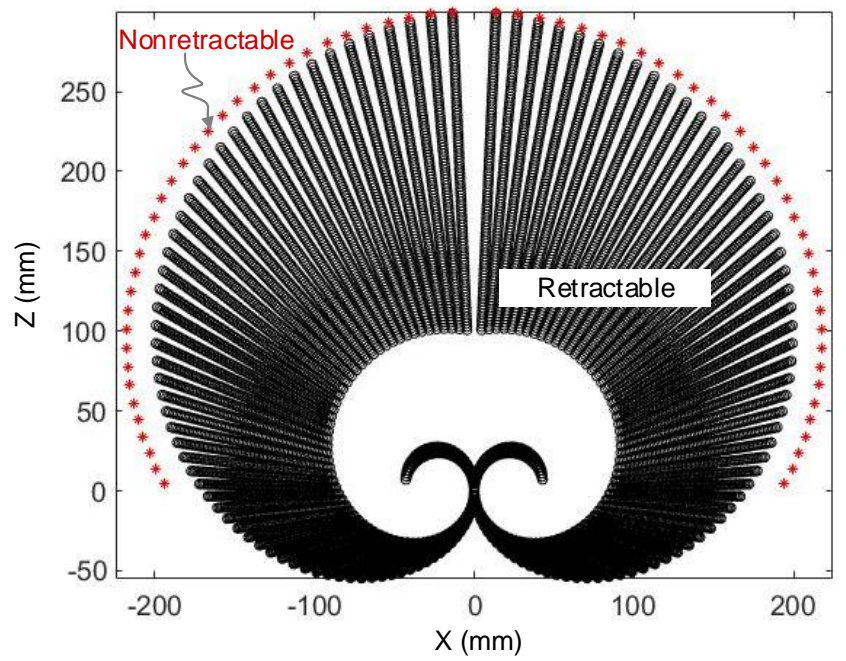

Fig. 4 The cut-viewed workspace of the robotic system with and without contractile ability.

From the above Fig. 4, we can know that the retractable ability enlarged the workspace significantly. The workspace of the former is a solid sphere-like with a small hole, but the latter is just a surface similar to that of a sphere. (Note that both of their workspace boundaries are not standard circular curves in cut view.)

\section{MOTION TEST OF THE PROTOTYPE}

Here we simply show the motion flexibility and workspace in Fig. 5. Besides, the contractile/extensive ability of the robotic arm is shown in Fig. 6. 


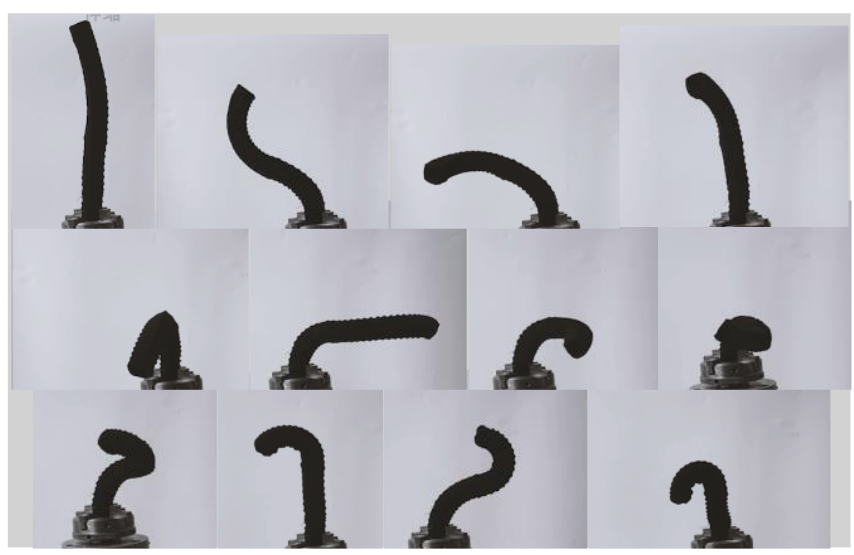

Fig. 5 The motion of the robotic system. These figures show the flexible omnidirectional bending ability and large bending curvature.

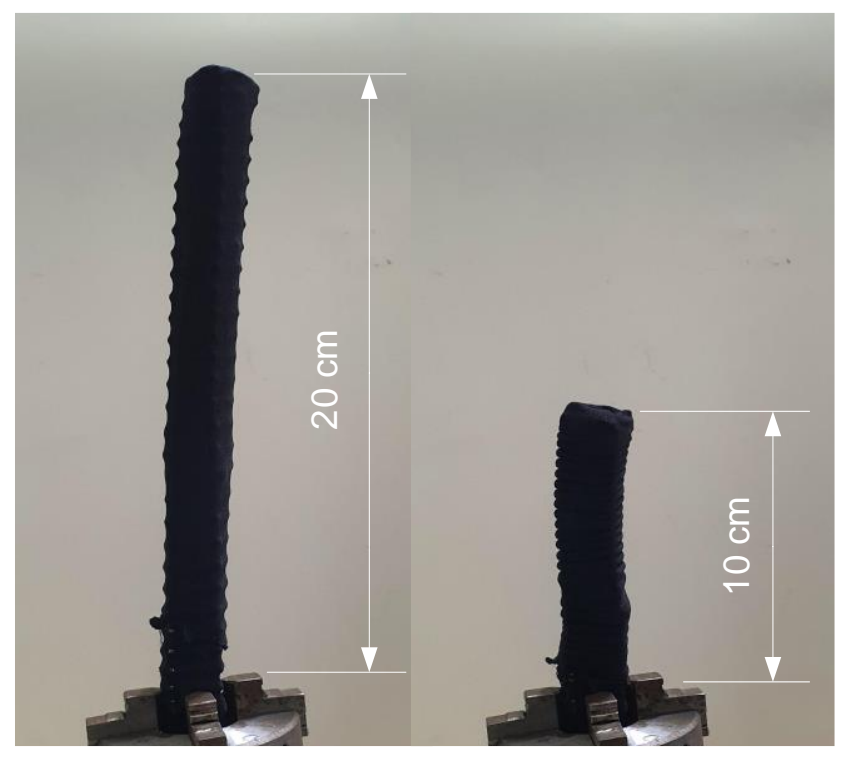

Fig. 6 The contractile ability of the robotic arm.

\section{DISCUSSION}

In this paper, we designed a novel continuum manipulator based on a contractile elastic mechanical spring-like skeleton. It exhibits advantages in the workspace that those continuum manipulators without contractile. With our mathematical model, a robotic arm with the required workspace can be analytically designed.

However, the workspace analysis in the current work is about that of a one-segment arm. This is mainly because that the ergodicity algorithm costs huge computation time. Manipulators with two segments will reduce the gap of workspace between the manipulators with and without contractile ability, i.e., the contractibility gives the manipulators more pose dexterities $\left(\theta_{x}, \theta_{y}, \theta_{z}\right)$ in a certain directional coordinate $(x, y, z)$ than ones without contractibility. We will study the workspace of the twosegment manipulator in the future.
Moreover, in this paper, we did not test the trajectory tracking of the manipulator because we yet have not established the physical controller which is essential for how to actuate the motor. There is much engineering work to do.

The payload ability of the proposed robotic arm, depending on the stiffness, should be discussed here. The payload can significantly influence the application range of the robot. This design makes its body softer in axis-direction than those manipulators with NiTi alloy skeleton or flexible rods instead of the tendons. However, the stiffness deeply depends on the materials and size parameters of the elastic skeleton. The design of the stiffness is also future work.

\section{CONCLUSION}

In the work, we designed a novel continuum manipulator with a contractile, tubular body. It only weighs about 40 grams; the body length can vary from about $10 \mathrm{~cm}$ to $30 \mathrm{~cm}$. The prototype and demos of the manipulator were performed. The demos and workspace analysis shows the advantages of the proposed design. The flexibility of motions and the diversified choices of materials enables the potential applications on detection in nuclear equipment, high-voltage transformers, aircraft engines; grasping in a greenhouse and underwater plants; transformation of liquid/gas drug or wastewater; and so on.

\section{ACKNOWLEDGMENTS}

This work was supported in part by the National Natural Science Foundation of China (Grant No. 91648106), and thanks for the scholarship of the Harbin Institute of Technology Scholarship Fund.

\section{REFERENCES}

[1] Zheng Li, Liao Wu, Hongliang Ren, and Haoyong Yu, "Kinematic comparison of surgical tendon-driven manipulators and concentric tube manipulators,"

[2] C. Chautems, A. Tonazzini, Q. Boehler, S. H. Jeong, D. Floreano, and B. J. Nelson, "Magnetic Continuum Device with Variable Stiffness for Minimally Invasive Surgery," Advanced Intelligent Systems, vol. 2, no. 6, p. 1900086, 2020, doi: 10.1002/aisy.201900086.

[3] B. Mazzolai, L. Margheri, M. Cianchetti, P. Dario, and C. Laschi, "Soft-robotic arm inspired by the octopus: II. From artificial requirements to innovative technological solutions," Bioinspiration \& Biomimetics, vol. 7, no. 2, p. 25005, 2012, doi: 10.1088/17483182/7/2/025005.

[4] T.-D. Nguyen and J. Burgner-Kahrs, "A tendon-driven continuum robot with extensible sections," in 2015 IEEE/RSJ International Conference on Intelligent Robots and Systems (IROS), Hamburg, Germany, Sep. 2015 - Oct. 2015, pp. 2130-2135.

[5] I. D. Walker, "Continuous Backbone "Continuum" Robot Manipulators," ISRN Robotics, vol. 2013, pp. 119, 2013, doi: 10.5402/2013/726506.

[6] H. Al-Fahaam, S. Nefti-Meziani, T. Theodoridis, and S. Davis, "The Design and Mathematical Model of a 
Novel Variable Stiffness Extensor-Contractor Pneumatic Artificial Muscle," Soft robotics, vol. 5, no. 5, pp. 576-591, 2018, doi: 10.1089/soro.2018.0010.

[7] D. C. Rucker and R. J. Webster III, "Statics and Dynamics of Continuum Robots With General Tendon Routing and External Loading," IEEE Trans. Robot., vol. 27, no. 6, pp. 1033-1044, 2011, doi: 10.1109/TRO.2011.2160469.

[8] Y. Yang and W. Zhang, "ET Arm: Highly Compliant Elephant-Trunk Continuum Manipulator," in Lecture Notes in Computer Science, Intelligent Robotics and Applications, X. Zhang, H. Liu, Z. Chen, and N. Wang, Eds., Cham: Springer International Publishing, 2014, pp. 288-299.

[9] K. Han, N.-H. Kim, and D. Shin, "A Novel Soft Pneumatic Artificial Muscle with High-Contraction Ratio," Soft robotics, vol. 5, no. 5, pp. 554-566, 2018, doi: 10.1089/soro.2017.0114.

[10] J. Burgner-Kahrs, H. B. Gilbert, J. Granna, P. J. Swaney, and R. J. Webster, "Workspace characterization for concentric tube continuum robots," in 2014 IEEE/RSJ International Conference on Intelligent Robots and Systems, Chicago, IL, USA, Sep. 2014 - Sep. 2014, pp. 1269-1275.

[11] Nabil Simaan, Russell Taylor, and Paul Flint, "LNCS 3217 - High Dexterity Snake-Like Robotic Slaves for Minimally Invasive Telesurgery of the Upper Airway,"

[12] L. H. Blumenschein, N. S. Usevitch, B. H. Do, E. W. Hawkes, and A. M. Okamura, "Helical actuation on a soft inflated robot body," in 2018 IEEE International Conference on Soft Robotics (RoboSoft), Livorno, Apr. 2018 - Apr. 2018, pp. 245-252.

[13] J. D. Greer, T. K. Morimoto, A. M. Okamura, and E. W. Hawkes, "A Soft, Steerable Continuum Robot That
Grows via Tip Extension," Soft robotics, vol. 6, no. 1, pp. 95-108, 2019, doi: 10.1089/soro.2018.0034.

[14] Hunter B. Gilbert and Robert James Webster III, "Can Concentric Tube Robots Follow the Leader?,"

[15] A. Garriga-Casanovas and F. Rodriguez y Baena, "Complete follow-the-leader kinematics using concentric tube robots," The International Journal of Robotics Research, vol. 37, no. 1, pp. 197-222, 2018, doi: 10.1177/0278364917746222.

[16] A. V. Kudryavtsev et al., “ Eye-in-Hand Visual Servoing of Concentric Tube Robots," IEEE Robot. Autom. Lett., vol. 3, no. 3, pp. 2315-2321, 2018, doi: 10.1109/LRA.2018.2807592.

[17] E. Amanov, T.-D. Nguyen, and J. Burgner-Kahrs, "Tendon-driven continuum robots with extensible sections - A model-based evaluation of path-following motions," The International Journal of Robotics Research, 027836491988604, 2019, doi: $10.1177 / 0278364919886047$.

[18] M. T. Chikhaoui, S. Lilge, S. Kleinschmidt, and J. Burgner-Kahrs, "Comparison of Modeling Approaches for a Tendon Actuated Continuum Robot With Three Extensible Segments," IEEE Robot. Autom. Lett., vol. 4, no. 2, pp. 989-996, 2019, doi: 10.1109/LRA.2019.2893610.

[19] G. Gao, H. Wang, J. Fan, Q. Xia, L. Li, and H. Ren, "Study on stretch-retractable single-section continuum manipulator," Advanced Robotics, vol. 33, no. 1, pp. 112, 2019, doi: 10.1080/01691864.2018.1554507.

[20] Guohua Gao, Hao Wang, Jingfang Liu, and Yuhang Zheng, "Statics analysis of an extensible continuum manipulator with large deflection," Mechanism and Machine Theory, 2019, 141:245-266. 